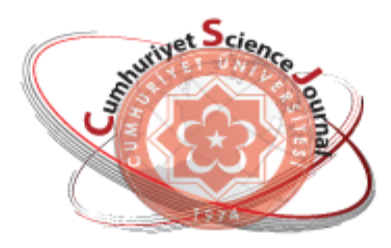

e-ISSN: 2587-246X

ISSN: $2587-2680$

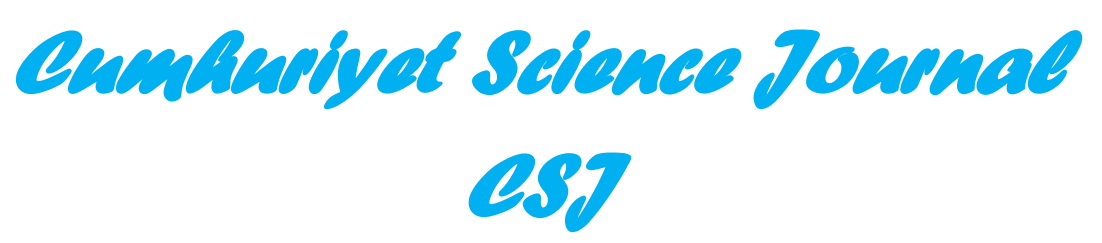

Cumhuriyet Sci. J., Vol.40-3 (2019) 570-577

\title{
Shell Model Calculations for Proton-rich Zn Isotopes via New Generated Effective Interaction by Artificial Neural Networks
}

\author{
Serkan AKKOYUN ${ }^{*}$ (D), Tuncay BAYRAM ${ }^{2}$ (D) \\ ${ }^{1}$ Department of Physics, Faculty of Science, Sivas Cumhuriyet University, Sivas, TURKEY \\ ${ }^{2}$ Department of Physics, Faculty of Science, Karadeniz Technical University, Trabzon, TURKEY
}

\begin{abstract}
In this study, the artificial neural network method has been employed for the generation of the new two-body matrix elements which is used for fpg shell nuclei. For this purpose, jj44b interaction Hamiltonian has been considered as a source. After the generation of the new Hamiltonian, both, original and new generated, are tested on proton-rich $\mathrm{Zn}$ isotopes. According to the results, the calculated values are close to the each other. As well the results from new interaction (jj44b_nn) are closer to the available experimental values in some cases.

Keywords: Nuclear shell model, Pfg shell, jj44b, Artificial neural network.

\section{Yapay Sinir Ağı ile Yeni Üretilen Etkin Etkileșimle Proton Zengini Zn İzotopları için Kabuk Modeli Hesaplamaları}

Özet. Bu çalışmada, fpg kabuk çekirdekleri için kullanılan iki cisim matris elemanlarının üretilmesi için yapay sinir ağı yöntemi kullanılmıştır. Bu amaçla, jj44b etkileşim Hamiltonian’i, kaynak olarak kabul edilmiştir. Yeni Hamiltonian'ın oluşumundan sonra, hem orijinal hem de yeni üretilen etkileşimin her ikisi de protonca zengin Zn izotopları üzerinde test edilmiştir. Elde edilen sonuçlara göre hesaplanan değerler birbirine yakındır. Ayrıca, yeni etkileşimden (jj44b_nn) elde edilen sonuçlar, mevcut deneysel değerlere ve literatür değerlerine daha yakın sonuç vermiştir.
\end{abstract}

Anahtar Kelimeler: Nükleer kabuk modeli, Pfg kabuğu, jj44b, Yapay sinir ağı.

\section{INTRODUCTION}

For the investigation of nuclear structure, nuclear shell model is a quite successful. Similar to the more familiar model for electronic shell model of atom, the nucleons are considered as located in the shells of a nucleus. If the number of nucleons equals to $2,8,2028,50,82$ or 126 which is named as nuclear magic number, the shells are said to be closed. The nuclei whose only one type nucleon number is magic are called magic nuclei. In the case of both types are magic, the nucleus is called as doubly-magic nuclei. In nuclear shell model (SM) calculations, a double-magic nucleus is considered as an inert core. The core nucleons do not interact with each other or the nucleons outside the core. Only valance nucleons above the closed shell are taken in the calculations. The space of the valance nucleons is a model space. 
The interactions of the nucleons in the model space with each other or the core are obtained via SM calculations [1-7]. Therefore, the correct estimations of the physical results in SM model, determination of the interaction Hamiltonian plays crucial role. In this paper, we have generated new interaction matrix elements from existed jj44b interaction [8]. This interaction is used for SM calculations of the nuclei outside the ${ }^{56} \mathrm{Ni}$ double-magic core and includes 133 two-body interaction matrix elements. The model space is pfg shell and contains the nuclei from mass number $A=56$ to 100. For the generation, we have applied artificial neural network (ANN) method which mimics the human brain function. Recently, ANN has been used in many fields of nuclear physics $[9,13]$. After generation of the new interaction matrix elements, the $2_{+}, 4_{+}$energies, $\mathrm{R}_{4 / 2}$ ratio, $\mathrm{B}(\mathrm{E} 2)$ values for proton-rich $\mathrm{Zn}$ isotopes have been calculated in the scope of SM. Kshell shell model code has been used which enables to perform nuclear shell-model calculations with M-scheme representation with the thick-restart Lanczos method [14].

The paper is organized as follows. In section 1, a brief introduction to the subject is given. In section 2, ANN method and shell model calculations have been summarized. Results of the ANN and the SM calculations for existed jj4b interaction and newly generated jj44b_nn interaction for $\mathrm{Zn}$ isotopes have been given in comparison with the available literature values in section 3. Finally, in the section 4, discussions on the obtained results have been given with the conclusions.

\section{MATERIAL and METHODS}

\subsection{Artificial Neural Network (ANN) Method}

A mathematical tool ANN mimics the human brain functionality [15]. The structure of ANN is composed of several processing units which are called neurons. The neurons are located in different layers and connected each other via adaptive synaptic weights. The first group of neurons is in input layer and receives data from outside. The data is transmitted to next group of neurons in hidden layer by weights. Finally, the processed data flows to the output layer neurons. In our calculation, we have used feedforward ANN with four layers in order to generate new two-body interaction matrix elements (tbme). The input layer consists of six neurons corresponding to a, b, c, d, J and T. The a, b, c, d values are the single particle orbits of the model space nucleons, $\mathrm{J}$ and $\mathrm{T}$ are total paired angular momentum and isospin, respectively. Due to the giving best results among the others, two hidden layers with 6 and 9 neurons in each are used as optimized value. The output layer neuron corresponds to the tbme. Therefore, the architecture of ANN is 6-6-9-1 and the total numbers of adjustable weights between neurons are 99 in this work. The hidden neuron activation function is tangent hyperbolic which is a sigmoid-like function generally used in the ANN calculations. For details of the ANN, the reader is referred to Haykin [15]. 


\subsection{Shell Model Calculation}

In the SM, valance nucleons move in a finite number of $\mathrm{j}$-orbits and their Hamiltonian of the valance nucleons is given by

$$
H=E_{0}+\sum_{i} \varepsilon_{i}+\sum_{a, b, c, d}<a b ; J T|V| c d ; J T>
$$

where $E_{0}$ is the energy of the inert core, $\varepsilon_{i}$ is single particle energies (spe) of the valance orbits and the last term $\langle a b ; J T|V| c d ; J T\rangle$ is two-body residual interaction among the valance particles. In the calculations with jj44b or jj44b_nn effective interactions, spe values are -9.2859, -9.6566, -8.2695 and 5.8944 for $\mathrm{p}_{3 / 2}, \mathrm{f}_{5 / 2}, \mathrm{p}_{1 / 2}$ and $\mathrm{g}_{9 / 2}$ shells. The valance nucleons are distributed in this pfg shells (Fig.1). The interaction Hamiltonian is defined by a set of 133 tbme. We have considered double magic ${ }^{56} \mathrm{Ni}$ isotope as a core whose proton and neutron numbers are 28 . The nucleons in the core with $\mathrm{J}=0$ do not move from the core.

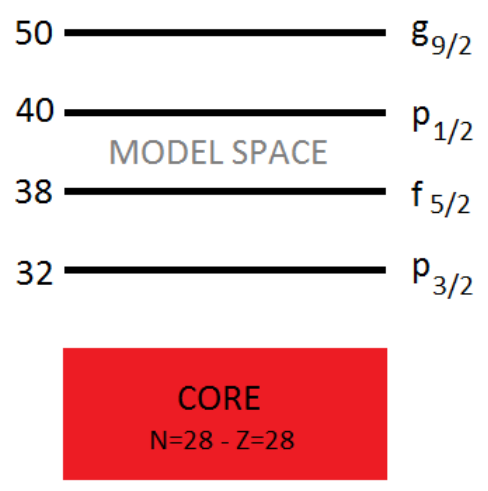

Figure 1 The fpg model space above the ${ }^{56} \mathrm{Ni}$ core for the calculations of $\mathrm{Zn}$ isotopes

There are many available codes in the literature which are written for the shell model calculations such as NuShell [16], Redstick [17], Bigstick [18], Antoine [19], Oxbash [20]. For the SM calculations for nuclear properties of proton-rich $\mathrm{Zn}$ isotopes, Kshell [14] computer code has been used in this present study. The code is a powerful computer code in order to calculate the energy levels, spins/parities, electric/magnetic quadrupole moments, electric/magnetic transition probabilities and one-particle spectroscopic factors in the nuclei. The code is used on a Linux operating system with a many-core CPU and OpenMP library. It is also used on a parallel computer with hybrid MPI+OpenMP parallel programming. If enough memory is available on the computers, up to tens of billions M-scheme dimension is capable. 


\section{RESULTS AND DISCUSSIONS}

In the first stage of the study, we have employed ANN to generate new interaction matrix elements from an existing data for the shell model calculations of fpg shell nuclei. By using constructed ANN mentioned in Section 2.1, we have obtained the elements whose values are not so far from the original ones. As can be seen in Table 1 that, the maximum difference between original and generated elements is 0.4861 for off-diagonal $\langle 41 ; 50|V| 42 ; 50\rangle$ tbme. The minimum value is 0 for several tbme. The more the hidden layer neurons the closest the result each other are. For the hidden neuron numbers 8-10, the constructed ANN starts to memorize the data and the generated tbme from it becomes to be same as the original tbme. Although the values are close each other for used ANN with 6-9 hidden neurons, the results of SM calculations have been improved by using new generated tbme. In order to see this improvement, SM calculations have been performed on proton-rich even-even $\mathrm{Zn}$ isotopes.

In Table 2, we have given the $2^{+}$and $4^{+}$energies of ${ }^{58-62} \mathrm{Zn}$ isotopes. For ${ }^{58} \mathrm{Zn}$, the theoretical calculated $2^{+}$energy values are same from jj44b and jj44b_nn. For ${ }^{60} \mathrm{Zn}$ isotope, $\mathrm{jj} 44 \mathrm{~b} \_n n$ gives results closer to the experimental value whereas for ${ }^{62} \mathrm{Zn}$ isotope, $\mathrm{jj} 44 \mathrm{~b}$ gives closer results. The calculations of $4^{+}$energy levels, jj44b_nn is better both for ${ }^{58} \mathrm{Zn}$ and ${ }^{60} \mathrm{Zn}$.

In Table 3, we have shown the B(E2) values for ground state to first $2^{+}$excited state and from $2^{+}$excited state to ground state. Only available experimental or adopted literature value is for ground state to $2^{+}$state in ${ }^{62} \mathrm{Zn}$ isotope. As is clear in the table that the jj44b_nn is slightly closer to the experimental value. For the other $\mathrm{Zn}$ isotopes, the results are very close to each other. In Table 4, we have listed the B(E2) values for first $2^{+}$excited state to first $4^{+}$excited state and from $4^{+}$excited state to $2^{+}$excited state. There is no available experimental or adopted value in the literature for these values. 
Table 1. Two-body matrix elements of jj44b and newly generated jj44b_nn interactions. The orbits are labeled by $1=p_{3 / 2}, 2=f_{5 / 2}$, $3=\mathrm{p}_{1 / 2}$ and $4=\mathrm{g}_{9 / 2}$ single particle orbits. The $\mathrm{a}, \mathrm{b}, \mathrm{c}, \mathrm{d}$ values are the single particle orbits, $\mathrm{J}$ and $\mathrm{T}$ are total paired angular momentum and isospin.

\begin{tabular}{|c|c|c|c|c|c|c|c|c|c|c|c|c|c|c|c|}
\hline $\mathbf{a}$ & b & c & d & $\mathbf{J}$ & $\mathbf{T}$ & jjj44b & jj44b_nn & $\mathbf{a}$ & b & c & d & $\mathbf{J}$ & $\mathbf{T}$ & jjj44b & jj44b_nn \\
\hline 1 & 1 & 1 & 1 & 1 & 0 & $-0,8113$ & $-0,8081$ & 4 & 4 & 4 & 4 & 9 & 0 & $-2,1260$ & $-2,1283$ \\
\hline 1 & 1 & 1 & 1 & 3 & 0 & $-0,4985$ & $-0,5162$ & 1 & 1 & 1 & 1 & 0 & 1 & $-1,3259$ & $-1,2921$ \\
\hline 1 & 1 & 1 & 1 & 5 & 0 & $-2,1319$ & $-2,1179$ & 1 & 1 & 1 & 1 & 2 & 1 & $-0,3349$ & $-0,3358$ \\
\hline 1 & 1 & 2 & 1 & 1 & 0 & 0,5205 & 0,5580 & 1 & 1 & 1 & 1 & 4 & 1 & 0,3138 & 0,3728 \\
\hline 1 & 1 & 2 & 1 & 3 & 0 & 0,3487 & 0,3125 & 1 & 1 & 2 & 1 & 2 & 1 & $-0,2903$ & $-0,2349$ \\
\hline 1 & 1 & 3 & 1 & 3 & 0 & $-0,6053$ & $-0,6000$ & 1 & 1 & 2 & 1 & 4 & 1 & $-0,4315$ & $-0,4270$ \\
\hline 1 & 1 & 2 & 2 & 1 & 0 & 0,1736 & 0,1988 & 1 & 1 & 3 & 1 & 2 & 1 & $-0,7133$ & $-0,6980$ \\
\hline 1 & 1 & 2 & 2 & 3 & 0 & 0,0320 & 0,0320 & 1 & 1 & 2 & 2 & 0 & 1 & $-0,7430$ & $-0,8444$ \\
\hline 1 & 1 & 3 & 2 & 1 & 0 & 0,1136 & $-0,0226$ & 1 & 1 & 2 & 2 & 2 & 1 & 0,0098 & 0,0499 \\
\hline 1 & 1 & 3 & 3 & 1 & 0 & $-0,2385$ & $-0,2908$ & 1 & 1 & 3 & 2 & 2 & 1 & 0,5004 & 0,4537 \\
\hline 1 & 1 & 4 & 4 & 1 & 0 & $-0,8790$ & $-0,8191$ & 1 & 1 & 3 & 3 & 0 & 1 & $-0,4773$ & $-0,4915$ \\
\hline 1 & 1 & 4 & 4 & 3 & 0 & $-0,3107$ & $-0,2993$ & 1 & 1 & 4 & 4 & 0 & 1 & 1,8722 & 1,8353 \\
\hline 1 & 1 & 4 & 4 & 5 & 0 & $-0,1703$ & $-0,1798$ & 1 & 1 & 4 & 4 & 2 & 1 & 0,2798 & 0,4341 \\
\hline 2 & 1 & 2 & 1 & 1 & 0 & $-1,9735$ & $-1,9779$ & 1 & 1 & 4 & 4 & 4 & 1 & 0,1879 & 0,0334 \\
\hline 2 & 1 & 2 & 1 & 2 & 0 & $-1,2445$ & $-1,2437$ & 2 & 1 & 2 & 1 & 1 & 1 & 0,2446 & 0,2890 \\
\hline 2 & 1 & 2 & 1 & 3 & 0 & $-0,6597$ & $-0,6621$ & 2 & 1 & 2 & 1 & 2 & 1 & 0,4202 & 0,3899 \\
\hline 2 & 1 & 2 & 1 & 4 & 0 & $-1,6087$ & $-1,6137$ & 2 & 1 & 2 & 1 & 3 & 1 & 0,5865 & 0,6269 \\
\hline 2 & 1 & 3 & 1 & 2 & 0 & $-0,7312$ & $-0,6938$ & 2 & 1 & 2 & 1 & 4 & 1 & $-0,1017$ & $-0,1027$ \\
\hline 2 & 1 & 3 & 1 & 3 & 0 & 0,6758 & 0,6572 & 2 & 1 & 3 & 1 & 2 & 1 & $-0,4233$ & $-0,3843$ \\
\hline 2 & 1 & 2 & 2 & 1 & 0 & 0,0665 & 0,0659 & 2 & 1 & 3 & 1 & 3 & 1 & 0,0290 & 0,0455 \\
\hline 2 & 1 & 2 & 2 & 3 & 0 & 0,3863 & 0,4129 & 2 & 1 & 2 & 2 & 2 & 1 & $-0,0657$ & $-0,1104$ \\
\hline 2 & 1 & 3 & 2 & 1 & 0 & 0,6218 & 0,6408 & 2 & 1 & 3 & 2 & 1 & 1 & 0,0725 & 0,0584 \\
\hline 2 & 1 & 3 & 2 & 2 & 0 & $-0,3687$ & $-0,4616$ & 2 & 1 & 3 & 2 & 2 & 1 & $-0,0633$ & $-0,1426$ \\
\hline 2 & 1 & 3 & 3 & 1 & 0 & 0,5089 & 0,6585 & 2 & 1 & 4 & 4 & 2 & 1 & 0,5475 & 0,5788 \\
\hline 2 & 1 & 4 & 4 & 1 & 0 & 0,3037 & 0,2474 & 2 & 1 & 4 & 4 & 4 & 1 & 0,5235 & 0,5430 \\
\hline 2 & 1 & 4 & 4 & 3 & 0 & $-0,0341$ & $-0,0234$ & 3 & 1 & 3 & 1 & 2 & 1 & $-0,1648$ & $-0,2051$ \\
\hline 3 & 1 & 3 & 1 & 2 & 0 & $-0,4481$ & $-0,4367$ & 3 & 1 & 3 & 1 & 3 & 1 & 0,6638 & 0,6220 \\
\hline 3 & 1 & 3 & 1 & 3 & 0 & $-1,4983$ & $-1,4898$ & 3 & 1 & 2 & 2 & 2 & 1 & $-0,2554$ & $-0,2632$ \\
\hline 3 & 1 & 2 & 2 & 3 & 0 & $-0,0006$ & 0,0032 & 3 & 1 & 3 & 2 & 2 & 1 & 0,4423 & 0,4458 \\
\hline 3 & 1 & 3 & 2 & 2 & 0 & $-0,5797$ & $-0,6143$ & 3 & 1 & 4 & 4 & 2 & 1 & 0,6139 & 0,5485 \\
\hline 3 & 1 & 4 & 4 & 3 & 0 & $-0,1910$ & $-0,1704$ & 4 & 1 & 4 & 1 & 2 & 1 & $-0,6032$ & $-0,6032$ \\
\hline 4 & 1 & 4 & 1 & 2 & 0 & $-3,5289$ & $-3,5389$ & 4 & 1 & 4 & 1 & 3 & 1 & $-0,0920$ & $-0,0419$ \\
\hline 4 & 1 & 4 & 1 & 3 & 0 & $-1,8098$ & $-1,8147$ & 4 & 1 & 4 & 1 & 4 & 1 & 0,4452 & 0,2406 \\
\hline 4 & 1 & 4 & 1 & 4 & 0 & $-1,2352$ & $-1,2256$ & 4 & 1 & 4 & 1 & 5 & 1 & 0,2804 & 0,4718 \\
\hline 4 & 1 & 4 & 1 & 5 & 0 & $-1,4659$ & $-1,4834$ & 4 & 1 & 4 & 1 & 6 & 1 & 0,6757 & 0,6048 \\
\hline 4 & 1 & 4 & 1 & 6 & 0 & $-0,8493$ & $-0,8040$ & 4 & 1 & 4 & 1 & 7 & 1 & $-0,7200$ & $-0,7734$ \\
\hline 4 & 1 & 4 & 1 & 7 & 0 & $-2,3659$ & $-2,4047$ & 4 & 1 & 4 & 2 & 3 & 1 & $-0,5352$ & $-0,4555$ \\
\hline 4 & 1 & 4 & 2 & 3 & 0 & $-0,8169$ & $-0,7536$ & 4 & 1 & 4 & 2 & 4 & 1 & $-0,1519$ & $-0,2064$ \\
\hline 4 & 1 & 4 & 2 & 4 & 0 & 0,5634 & 0,2266 & 4 & 1 & 4 & 2 & 5 & 1 & $-0,0907$ & 0,0130 \\
\hline 4 & 1 & 4 & 2 & 5 & 0 & $-0,1897$ & 0,2964 & 4 & 1 & 4 & 2 & 6 & 1 & $-0,4143$ & $-0,3692$ \\
\hline 4 & 1 & 4 & 2 & 6 & 0 & 0,8527 & 0,6543 & 4 & 1 & 4 & 3 & 4 & 1 & 0,1336 & 0,1116 \\
\hline 4 & 1 & 4 & 3 & 4 & 0 & $-0,8072$ & $-0,6775$ & 4 & 1 & 4 & 3 & 5 & 1 & $-0,0729$ & $-0,0952$ \\
\hline 4 & 1 & 4 & 3 & 5 & 0 & $-0,8831$ & $-0,9912$ & 2 & 2 & 2 & 2 & 0 & 1 & $-0,7572$ & $-0,7504$ \\
\hline 2 & 2 & 2 & 2 & 1 & 0 & $-0,8551$ & $-0,8573$ & 2 & 2 & 2 & 2 & 2 & 1 & 0,0862 & 0,1305 \\
\hline 2 & 2 & 2 & 2 & 3 & 0 & $-2,0525$ & $-2,0635$ & 2 & 2 & 3 & 2 & 2 & 1 & 0,2157 & 0,2559 \\
\hline 2 & 2 & 3 & 2 & 1 & 0 & $-1,3769$ & $-1,3686$ & 2 & 2 & 3 & 3 & 0 & 1 & $-1,1326$ & $-1,1276$ \\
\hline 2 & 2 & 3 & 3 & 1 & 0 & 0,6256 & 0,6214 & 2 & 2 & 4 & 4 & 0 & 1 & 0,9686 & 0,9654 \\
\hline 2 & 2 & 4 & 4 & 1 & 0 & 0,5998 & 0,5808 & 2 & 2 & 4 & 4 & 2 & 1 & 0,5567 & 0,4995 \\
\hline 2 & 2 & 4 & 4 & 3 & 0 & 0,4825 & 0,5337 & 3 & 2 & 3 & 2 & 1 & 1 & 0,3052 & 0,3135 \\
\hline 3 & 2 & 3 & 2 & 1 & 0 & $-2,3068$ & $-2,3085$ & 3 & 2 & 3 & 2 & 2 & 1 & $-0,3941$ & $-0,4021$ \\
\hline 3 & 2 & 3 & 2 & 2 & 0 & $-2,0031$ & $-1,9665$ & 3 & 2 & 4 & 4 & 2 & 1 & $-0,1474$ & $-0,0989$ \\
\hline 3 & 2 & 3 & 3 & 1 & 0 & $-0,3608$ & $-0,3664$ & 4 & 2 & 4 & 2 & 3 & 1 & $-0,8576$ & $-0,8774$ \\
\hline 3 & 2 & 4 & 4 & 1 & 0 & 0,5368 & 0,5290 & 4 & 2 & 4 & 2 & 4 & 1 & 0,1169 & 0,1576 \\
\hline 4 & 2 & 4 & 2 & 3 & 0 & $-0,9996$ & $-1,0071$ & 4 & 2 & 4 & 2 & 5 & 1 & $-0,1975$ & $-0,2201$ \\
\hline 4 & 2 & 4 & 2 & 4 & 0 & $-0,8777$ & $-0,8467$ & 4 & 2 & 4 & 2 & 6 & 1 & 0,8224 & 0,7916 \\
\hline 4 & 2 & 4 & 2 & 5 & 0 & $-0,3781$ & $-0,4948$ & 4 & 2 & 4 & 3 & 4 & 1 & 0,2570 & 0,2396 \\
\hline 4 & 2 & 4 & 2 & 6 & 0 & $-2,3615$ & $-2,3469$ & 4 & 2 & 4 & 3 & 5 & 1 & $-0,4919$ & $-0,4556$ \\
\hline 4 & 2 & 4 & 3 & 4 & 0 & 1,0058 & 0,9069 & 3 & 3 & 3 & 3 & 0 & 1 & $-0,1139$ & $-0,1060$ \\
\hline 4 & 2 & 4 & 3 & 5 & 0 & $-0,3069$ & $-0,1848$ & 3 & 3 & 4 & 4 & 0 & 1 & 0,8110 & 0,8110 \\
\hline 3 & 3 & 3 & 3 & 1 & 0 & $-1,0199$ & $-1,0155$ & 4 & 3 & 4 & 3 & 4 & 1 & 0,3293 & 0,3363 \\
\hline 3 & 3 & 4 & 4 & 1 & 0 & $-0,2545$ & $-0,2517$ & 4 & 3 & 4 & 3 & 5 & 1 & $-0,2067$ & $-0,2205$ \\
\hline 4 & 3 & 4 & 3 & 4 & 0 & $-1,6832$ & $-1,7087$ & 4 & 4 & 4 & 4 & 0 & 1 & $-1,4086$ & $-1,4040$ \\
\hline 4 & 3 & 4 & 3 & 5 & 0 & $-1,1173$ & $-1,1116$ & 4 & 4 & 4 & 4 & 2 & 1 & $-1,0366$ & $-1,0421$ \\
\hline 4 & 4 & 4 & 4 & 1 & 0 & $-1,0265$ & $-1,0103$ & 4 & 4 & 4 & 4 & 4 & 1 & $-0,2288$ & $-0,2259$ \\
\hline 4 & 4 & 4 & 4 & 3 & 0 & $-0,5628$ & $-0,5201$ & 4 & 4 & 4 & 4 & 6 & 1 & 0,2066 & 0,1920 \\
\hline 4 & 4 & 4 & 4 & 5 & 0 & $-0,5590$ & $-0,6344$ & 4 & 4 & 4 & 4 & 8 & 1 & 0,2457 & 0,2489 \\
\hline 4 & 4 & 4 & 4 & 7 & 0 & $-0,8680$ & $-0,8583$ & & & & & & & & \\
\hline
\end{tabular}


Table 2. First 2+ and 4+ energies of proton rich $\mathrm{Zn}$ isotopes

\begin{tabular}{|lllllll|}
\hline & \multicolumn{3}{c}{$2^{+}$Energy $(\mathrm{keV})$} & & $4^{+}$Energy $(\mathrm{keV})$ \\
\hline Isotope & $\exp$ & $j j 4 b$ & $j j 4 b \_n n$ & $\exp$ & $j j 4 b$ & $j j 4 b \_n n$ \\
\hline${ }^{58} \mathbf{Z n}$ & 1356 & 1683 & 1683 & 2499 & 2299 & 2323 \\
\hline${ }^{60} \mathbf{Z n}$ & 1004 & 998 & 1001 & 2193 & 2112 & 2139 \\
\hline${ }^{62} \mathbf{Z n}$ & 954 & 958 & 960 & 2186 & 2220 & 2238 \\
\hline
\end{tabular}

Table 3. B(E2) values between ground state and first $2+$ state for proton rich $\mathrm{Zn}$ isotopes

\begin{tabular}{|ccccccc|}
\hline Isotope & $\exp$ & $\mathrm{B}(\mathrm{E} 2 ; 0-2)$ & & & $\mathrm{B}(\mathrm{E} 2 ; 2-0)$ & \\
\hline${ }^{58} \mathbf{Z n}$ & - & 466.87 & 467.41 & - & 93.37 & 93.48 \\
\hline${ }^{60} \mathbf{Z n}$ & - & 709.39 & 710.86 & - & 141.88 & 142.17 \\
\hline${ }^{62} \mathbf{Z n}$ & 1240 & 807.99 & 809.55 & - & 161.60 & 161.91 \\
\hline
\end{tabular}

Table 4. B(E2) values between first $2+$ state and $4+$ state for proton rich $\mathrm{Zn}$ isotopes

\begin{tabular}{|ccccccc|}
\hline Isotope & $\exp$ & $\mathrm{B}(\mathrm{E} 2 ; 2-4)$ & $j j 4 b \_n n$ & $\exp$ & $j j 4 b$ & $j j 4 b \_n n$ \\
\hline${ }^{58} \mathbf{Z n}$ & - & 155.68 & 155.52 & - & 86.49 & 86.40 \\
\hline${ }^{60} \mathbf{Z n}$ & - & 308.5 & 309.08 & - & 171.39 & 171.71 \\
\hline${ }^{62} \mathbf{Z n}$ & - & 373.89 & 375.27 & - & 207.72 & 208.49 \\
\hline
\end{tabular}

We have also calculated $\mathrm{R}_{4 / 2}$ and $\mathrm{B}(\mathrm{E} 2 ; 4$->2)/B(E2;2->0) ratios for $\mathrm{Zn}$ isotopes. In Fig.2, we have shown the $\mathrm{R}_{4 / 2}$ ratios from experimental results and theoretical results by jj44b and jj44b_nn interactions. It is clear in figure that, the results from newly generated jj44b_nn tbme are slightly closer to the experimental data for ${ }^{58-60} \mathrm{Zn}$ isotopes.

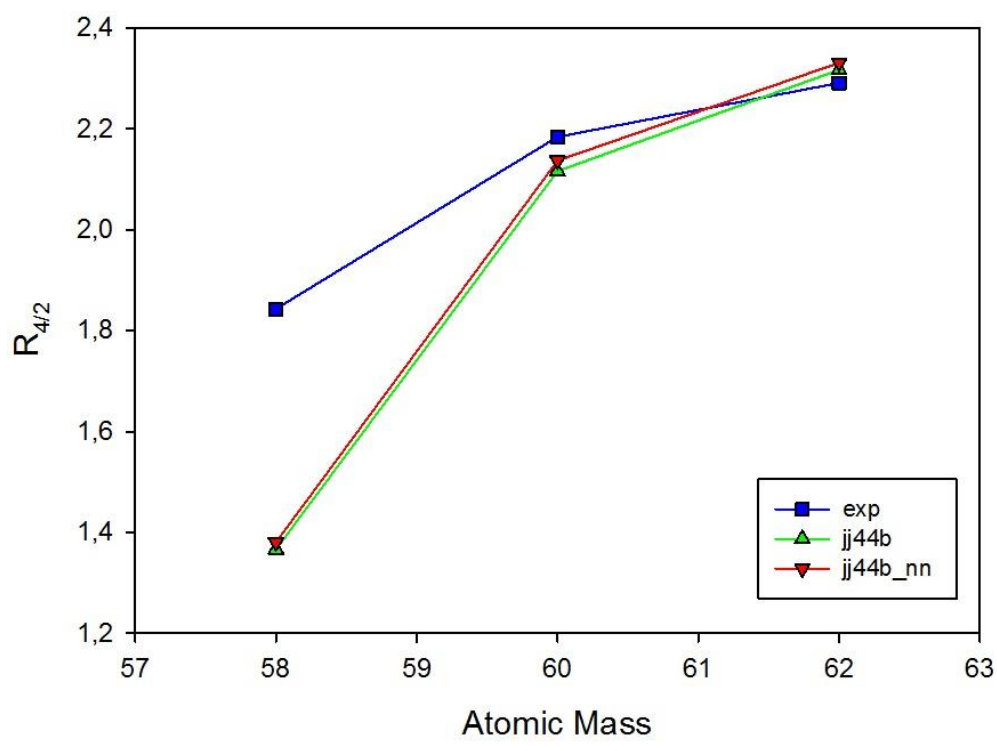

Figure. $2 \mathrm{R}_{4 / 2}$ values for proton-rich $\mathrm{Zn}$ isotopes. 
Finally in Fig.3, we have shown the $\mathrm{B}(\mathrm{E} 2 ; 4->2) / \mathrm{B}(\mathrm{E} 2 ; 2->0)$ ratios from experimental results and theoretical results by jj44b and jj44b_nn interactions. Only available experimental values are for ${ }^{62} \mathrm{Zn}$ isotope with the value of 1.55 . Neither $\mathrm{jj} 44 \mathrm{~b}$ nor $\mathrm{jj} 44 \mathrm{~b} \_n n$ results are close to this value. The results from both calculations is almost same each other.

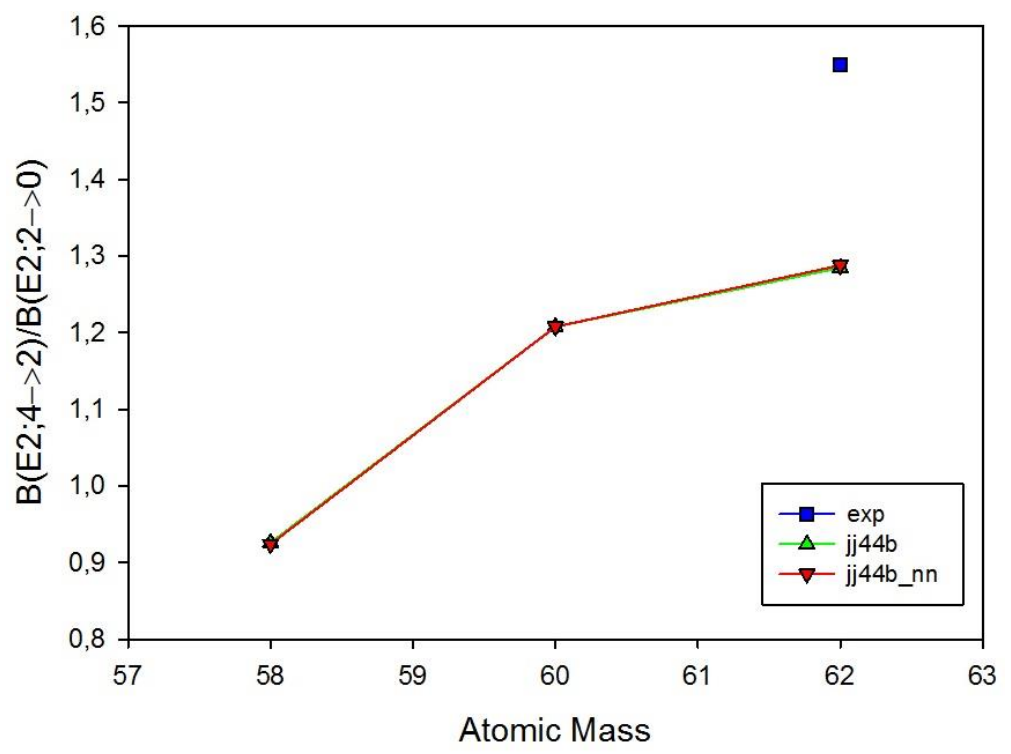

Figure. $3 \mathrm{~B}(\mathrm{E} 2 ; 4->2) / \mathrm{B}(\mathrm{E} 2 ; 2->0)$ values for proton-rich $\mathrm{Zn}$ isotopes.

\section{Acknowledgements}

This work is supported by the scientific research project fund of Sivas Cumhuriyet University under the project number SHMYO-015.

\section{REFERENCES}

[1] Mayer, M.G., On Closed Shells in Nuclei. II, Phys.Rev. 75 (1949) 1969.

[2] Jensen, J.H.D., et al.,On the "Magic Numbers" in Nuclear Structure, Phys.Rev. 75 (1949) 1766.

[3] Mayer, M.G., On Closed Shells in Nuclei, Phys.Rev. 74 (1948) 235.

[4] Mayer, M.G., Nuclear Configurations in the Spin-Orbit Coupling Model. I. Empirical Evidence, Phys.Rev. 78 (1950) 16.

[5] Talmi, I., 55 Years Of The Shell Model: A Challenge To Nuclear Many-Body Theory, Int.J.Mod.Phys.E 14 (2005) 821.

[6] Caurier, E., etal., The shell model as a unified view of nuclear structure, Rev.Mod.Phy. 77 (2005) 427.

[7] Brown, B.A., The nuclear shell model towards the drip lines, Prog.Part.Nucl.Phys. 47 (2001) 517.

[8] Brown, B.A. and Lisetskiy, A.F., unpublished. The jj44b Hamiltonian was obtained from a fit to about 600 binding energies and excitation energies with a method similar to that used for the JUN45 Hamiltonian. 
[9] Neufcourt, L., et al., Bayesian approach to model-based extrapolation of nuclear observables, Phys.Rev. C 98 (2018) 034318.

[10] Negoita, G.A., et al., Deep Learning: A Tool for Computational Nuclear Physics, arXiv:1803.03215 [physics.comp-ph] (2018).

[11] Yildiz, N., et al., Consistent Empirical Physical Formula Construction for Gamma Ray Angular Distribution Coefficients by Layered Feedforward Neural Network, Cumhuriyet Sci.J., 39 (2018) 928.

[12] Bayram, T., et al., A study on ground-state energies of nuclei by using neural networks, Ann.Nucl.Energy., 63 (2014) 172.

[13] Akkoyun, S., et al., An artificial neural network application on nuclear charge radii, J. Phys. G: Nucl. Part. Phys., 40 (2013) 055106.

[14] Shimizu, N. Nuclear shell-model code for massive paralel computation, KSHELL, arXiv:1310.5431 [nucl-th] (2013).

[15] Haykin, S., Neural Networks: A Comprehensive Foundation (Englewood Cliffs, NJ: Prentice-Hall) (1999).

[16] Brown, B.A., Rae, W.D.M., The Shell-Model Code NuShellX@MSU Nucl.Data Sheets. 120 (2014) 115.

[17] REDSTICK, http://www.phys.lsu.edu/faculty/cjohnson/redstick.html

[18] Jhonson, C.W., et al., BIGSTICK: A flexible configuration-interaction shell-model code, arXiv:1801.08432v1 [physics.comp-ph] (2018).

[19] ANTOINE, http://www.iphc.cnrs.fr/nutheo/code_antoine/menu.html

[20] B. A. Brown, et al., Oxbash for Windows, MSU_NSCL report number 1289. 